\title{
ARTICLE
}

DOI: $10.1038 /$ ncomms10971

OPEN

\section{Different states of synaptotagmin regulate evoked versus spontaneous release}

Hua Bai ${ }^{1, \star}, \uparrow$, Renhao Xue ${ }^{1, \star}$, Huan Bao ${ }^{1}$, Leili Zhang ${ }^{2}$, Arun Yethiraj ${ }^{2}$, Qiang Cui ${ }^{2} \&$ Edwin R. Chapman ${ }^{1}$

The tandem C2-domains of synaptotagmin 1 (syt) function as $\mathrm{Ca}^{2+}$-binding modules that trigger exocytosis; in the absence of $\mathrm{Ca}^{2+}$, syt inhibits spontaneous release. Here, we used proline linkers to constrain and alter the relative orientation of these C2-domains. Short poly-proline helices have a period of three, so large changes in the relative disposition of the C2-domains result from changing the length of the poly-proline linker by a single residue. The length of the linker was varied one residue at a time, revealing a periodicity of three for the ability of the linker mutants to interact with anionic phospholipids and drive evoked synaptic transmission; syt efficiently drove exocytosis when its tandem C2-domains pointed in the same direction. Analysis of spontaneous release revealed a reciprocal relationship between the activation and clamping activities of the linker mutants. Hence, different structural states of syt underlie the control of distinct forms of synaptic transmission.

\footnotetext{
${ }^{1}$ Howard Hughes Medical Institute and Department of Neuroscience, University of Wisconsin, Madison, Wisconsin 53705, USA. ${ }^{2}$ Department of Chemistry and Theoretical Chemistry Institute, University of Wisconsin, Madison, Wisconsin 53706, USA. * These authors contributed equally to this work. $\dagger$ Present address: Department of Biochemistry, University of Washington, Seattle, Washington 98195, USA. Correspondence and requests for materials should be addressed to E.R.C. (email: chapman@wisc.edu).
} 
E voked neurotransmitter release mediates rapid communication between neurons, and thus underlies neural network function ${ }^{1}$. Spontaneous neurotransmitter release also occurs, and this mode of secretion affects myriad aspects of synaptic function including post-synaptic protein synthesis and the maintenance of synaptic contacts ${ }^{2,3}$. Synaptotagmin 1 (syt) is an abundant integral membrane protein that is targeted to synaptic vesicles (SVs) where it controls both of these forms of exocytosis, by serving as a $\mathrm{Ca}^{2+}$ sensor that triggers evoked release, and by acting as a fusion clamp that prevents exocytosis under resting conditions ${ }^{4-7}$. It is not known how syt can switch states from an inhibitor to an activator of secretion. This is a puzzling problem, as syt senses $\mathrm{Ca}^{2+}$ via tandem $\mathrm{C} 2$-domains, $\mathrm{C} 2 \mathrm{~A}$ and $\mathrm{C} 2 \mathrm{~B}$, which comprise most of the cytoplasmic domain of the protein, and these isolated domains do not exhibit significant conformational changes upon binding metal ${ }^{8,9}$.

Syt interacts with soluble $N$-ethylmaleimide-sensitive factor (NSF) attachment protein receptors (SNAREs) ${ }^{10}$, which form complexes that are both necessary and sufficient for membrane fusion ${ }^{11}$. In neurons, the SNARE complex consists of the vesicular (v-) SNARE, synaptobrevin (syb), and the target membrane ( $\mathrm{t}-$ ) SNAREs, syntaxin 1 (syx) and SNAP-25. The assembly of $\mathrm{v}$ - and $\mathrm{t}$-SNAREs into SNARE complexes provides sufficient energy to drive slow rates of membrane fusion in vitro ${ }^{12}$. Syt binds directly to t-SNAREs, but not to v-SNAREs, and inclusion of syt in reconstituted fusion assays renders SNARE catalysed fusion reactions dependent on calcium ions ${ }^{13}$. Additional biochemical studies revealed that $\mathrm{Ca}^{2+} \bullet$ syt directly regulates the assembly of SNARE complexes ${ }^{14-16}$. However, cell-based experiments provide evidence both for and against the idea that syt॰t-SNARE interactions trigger fusion, and a consensus has not been reached ${ }^{4,17-20}$.

Syt also directly interacts with membranes that harbour anionic phospholipids ${ }^{21}$. Upon binding $\mathrm{Ca}^{2+}$, the distal tips of $\mathrm{Ca}^{2+}$-binding loops 1 and 3 (see Fig. 1 for details) in both C2A and $\mathrm{C} 2 \mathrm{~B}$ partially insert into the target membrane, ${ }^{4,22-24}$, potentially generating localized curvature $e^{25,26}$ and/or pulling the two membranes together to mediate close apposition ${ }^{15,23,27}$, thereby facilitating fusion. Both in vitro and cell-based experiments indicate that $\mathrm{Ca}^{2+} \bullet$ syt $\bullet$ membrane interactions constitute a crucial step in excitation-secretion coupling ${ }^{4,28}$.

The tandem C2-domains of syt are connected by a nine-residue linker that is flexible, and might allow the C2-domains to adopt multiple distinct relative orientations ${ }^{29-32}$. The objective of the current study was to constrain these C2-domains to begin to determine whether changes in their relative configuration underlie the ability of syt to change functional states, from a clamp to an activator of fusion. This question was addressed by exploiting the properties of poly-proline segments. Proline is a unique amino acid; its side-chain merges with its backbone, thus restricting the backbone dihedral angles to a limited range ${ }^{33,34}$. These properties underlie the finding that short poly-proline segments form rigid helices with a periodicity of three (note: the structures of longer poly-proline motifs, beyond 12-15 residues, can be complex because of the increasing probability of introducing cis proline conformations ${ }^{35}$ ). Because of this rigidity, short poly-proline rods have been used as spectroscopic rulers, or rigid spacers, for decades ${ }^{34-37}$. However, the periodicity of these helices has not been extensively exploited to address protein function and engineering problems.

Here, we replaced the native nine-residue linker of syt with a nine-residue poly-proline rod, and confirmed an earlier study demonstrating that this mutant is functional in terms of driving fusion in both in vitro and cell-based assays ${ }^{4}$. Interestingly, molecular dynamics (MD) simulations predicted that the C2-domains in this mutant form of syt were not only highly constrained, but also have a strong tendency to point in the same direction, likely towards the plasma membrane. We then varied the length of the linker, one residue at a time, to systematically alter the relative angle between the tandem $\mathrm{C} 2$-domains, and observed a clear periodicity of three regarding the activity of these

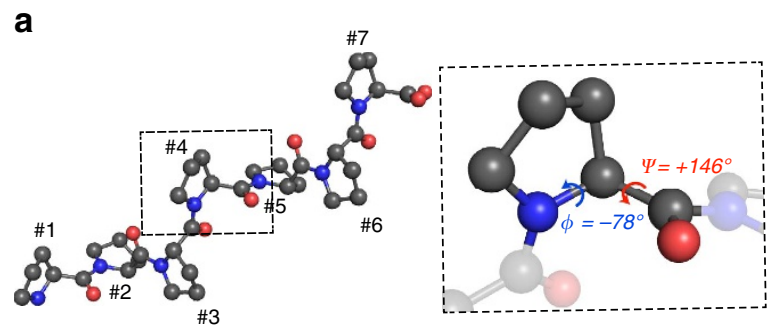

b
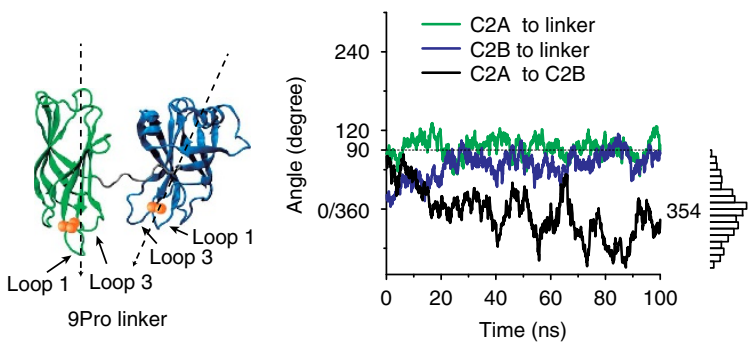

C

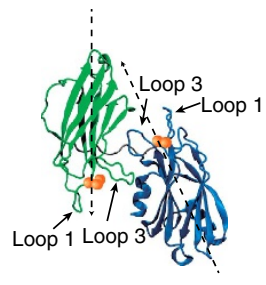

10Pro linker

d

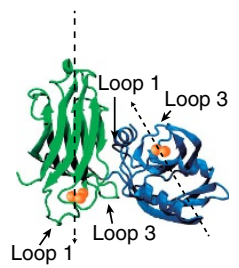

11Pro linker
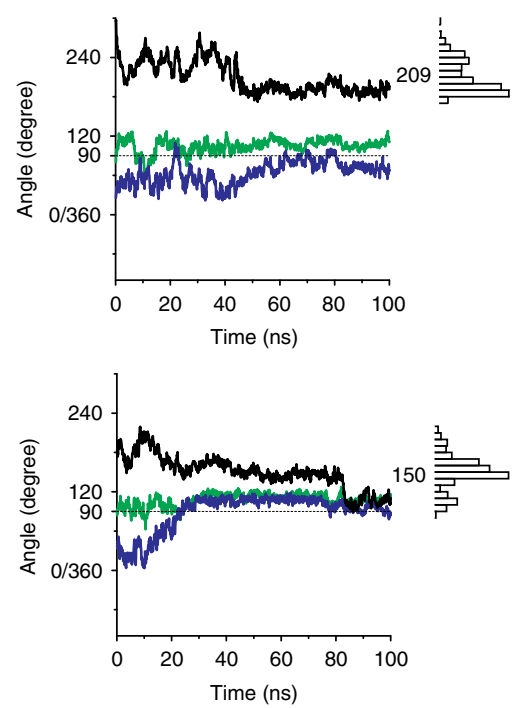

Figure 1 | Constraining the relative orientation between the C2A and C2B domains of syt using poly-proline linkers. (a) A seven-residue poly-proline (7Pro) helical rod is shown in the left panel; the pitch is three residues per turn of the helix. Right panel: Magnified view in which the dihedral angels, which underlie the periodicity of three, are shown. Nitrogen atoms are represented with blue balls, and the oxygen atoms are represented in Red. (b-d) The relative orientations of $\mathrm{C} 2 \mathrm{~A}$ and $\mathrm{C} 2 \mathrm{~B}$ are predicted to be constrained, and thus altered, by varying the number of residues in the poly-proline linker; these findings are based on the analysis of the $\mathbf{1 a - 2} \mathbf{b}$ conformer (see definitions and discussion in Supplementary Note 1, Supplementary Figs 7-9 and Supplementary Table 8). Left panels show representative snapshots for the tandem C2-domains connected by polyproline linkers consisting 9 (b), 10 (c) or 11 (d) residues. Right panels: Results from MD simulations, revealing the predicted relative C2-domain orientations as a function of time. The green and blue traces indicate the relative orientation between the proline linker and either $\mathrm{C} 2 \mathrm{~A}$ or $\mathrm{C} 2 \mathrm{~B}$, respectively. Black traces indicate the relative orientation between $C 2 A$ and $\mathrm{C} 2 \mathrm{~B}$; histograms and numerical averages of relative orientation angles are provided on the right side of each panel. 
mutants in a variety of biochemical and functional assays. These observations, in conjunction with the results from photoinduced electron transfer (PET) quenching experiments, demonstrate that the C2-domains were in fact constrained to specific, discernable, orientations. Importantly, there was a clear reciprocal relationship between the abilities of the linker mutants to clamp spontaneous release and to drive evoked release: mutants that drove efficient evoked release failed to inhibit spontaneous release and visa-versa. Hence, syt switches states, from inhibitor to activator of fusion, via dynamic alterations in the relative orientation of its tandem C2-domains.

\section{Results}

Computational modelling of poly-proline linker mutants. The major goal of this study was to fix the relative orientation of the $\mathrm{C} 2$-domains of syt into specific states, and to determine whether different orientations subserve distinct functions of the protein. To address this, we first replaced the native linker that connects these domains (residues 264-272, SAEKEEQEK) with a nine-residue poly-proline segment (9Pro). As outlined above, poly-proline was used because the side chain of this residue merges into its backbone, so the dihedral angles are relatively stable, with $\Phi=-78^{\circ}$ and $\Psi=+146^{\circ}$, resulting in a rigid helix with a periodicity of three $33-35$ (Fig. 1a).

We next sought to determine whether the C2-domains were free to rotate around the proline linker, or whether they were fixed relative to the linker, and hence fixed relative to one-another. We first addressed this question via all-atom MD simulations and metadynamics analysis (detailed in the Methods and Supplementary Notes 1 ). This analysis revealed that C2A and $\mathrm{C} 2 \mathrm{~B}$ are highly constrained with respect to the poly-proline linker, and that $\mathrm{Ca}^{2+}$-binding loops of the $\mathrm{C} 2 \mathrm{~A}$ and $\mathrm{C} 2 \mathrm{~B}$ tend to 'point' in roughly the same direction in 9Pro mutant form of syt $\left(354^{\circ}\right.$; Fig. 1b). These findings made it possible to systematically alter the relative orientation of these domains by altering the linker one residue at a time, as adding or removing a single residue should have large effects. This prediction was confirmed by simulations using proline linkers of 10 and 11 residues, where the relative angle between $\mathrm{C} 2 \mathrm{~A}$ and $\mathrm{C} 2 \mathrm{~B}$ shifted to $209^{\circ}$ and $150^{\circ}$, respectively (Fig. 1c,d). Hence, for biochemical and functional studies, we varied the linker from 6 to 9 , or in some cases up to 12, prolines (6Pro to 12Pro). Further below, we describe PET experiments that confirm that when the linker is in multiples of three prolines, $\mathrm{C} 2 \mathrm{~A}$ and $\mathrm{C} 2 \mathrm{~B}$ point in the same direction.

Comparison of syt linker mutants in in vitro fusion assays. We examined the impact of the poly-proline linkers on the function of syt using a 'split t-SNARE' version of the reconstituted, SNARE-mediated, membrane fusion assay ${ }^{14,15}$ (Fig. 2a). Briefly, syx was reconstituted, but the other t-SNARE, SNAP-25, was added in a soluble form, such that $\mathrm{Ca}^{2+} \bullet$ syt must fold SNAP-25 onto membrane-embedded syx to drive fusion. Optimal folding activity requires $\mathrm{Ca}^{2+} \bullet$ syt $\bullet$ membrane interactions ${ }^{14}$, so this assay system involves both syt-membrane interactions as well as syt-mediated assembly of SNARE complexes. For these, and all biochemical experiments reported here, the cytoplasmic domain of syt was used, and for the electrophysiological studies detailed further below, full-length syt was used.

Upon addition of $\mathrm{Ca}^{2+}$, fusion was accelerated by wild-type (WT) syt and each of the linker mutants, but clear differences among the mutants were apparent (Fig. 2b,c). Notably, 6Pro, 9Pro and 12Pro exhibited the greatest activity, followed by 8 Pro and 11 Pro, whereas 7 Pro and 10Pro were the least effective at stimulating fusion. These data were quantified and plotted in Fig. 2c; remarkably, the extent of fusion was well fitted by a wave
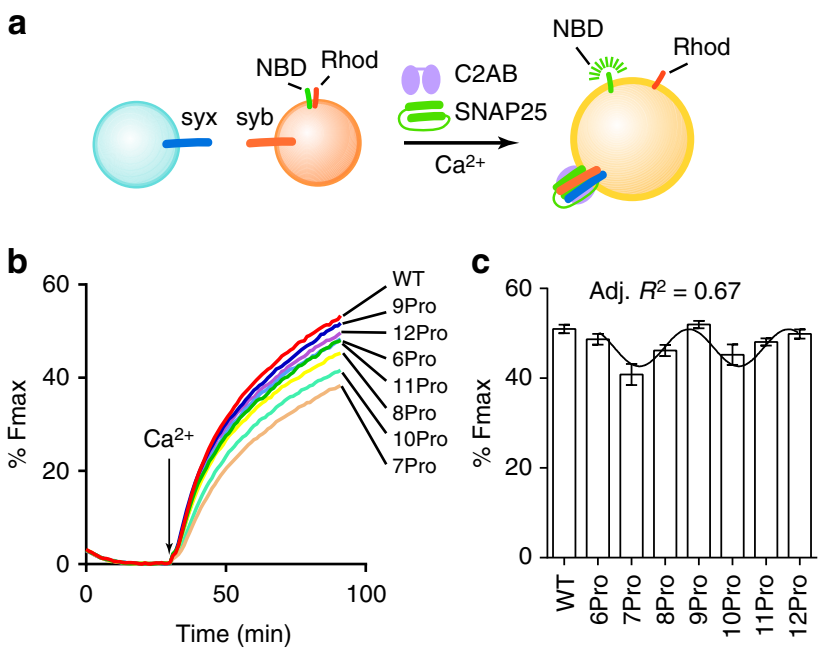

Figure 2 | Analysis of syt linker mutant activity in reconstituted fusion reactions. (a) Schematic diagram of the reconstituted fusion system; the fluorescence resonance energy transfer donor (NBD) fluorescence increases, because of dequenching, upon fusion. (b) Representative traces of reconstituted membrane fusion reactions regulated by WT or linker mutant forms of syt $(3 \mu \mathrm{M})$; the arrow indicates the addition of $\mathrm{Ca}^{2+}$ $\left(1 \mathrm{mM}\right.$, free). (c) The extent of fusion $1 \mathrm{~h}$ after addition of $\mathrm{Ca}^{2+}$ was plotted and found to be periodic, with a period of three. Data are represented as mean \pm s.e.m. For each condition, three independent trials were carried out. Data were fitted using a sine wave function with a periodicity of three; an adjusted (Adj.) $R^{2}$ value was generated to assess the goodness of the fit.

function with a periodicity of three. This periodicity is consistent with the periodicity of poly-proline rods. Combined with the MD simulations, these results indicate that the ability of syt to drive membrane fusion is strongly influenced by the relative orientation of the tandem C2-domains, and that specific orientations are needed to effectively regulate membrane fusion. This is not a length issue, as the proline rod is fully functional in 12Pro; length becomes an issue when the rod is extended out to 18 prolines, as reported previously ${ }^{4}$. Again, because syt regulates fusion via interactions with both anionic phospholipids ${ }^{4,28,38}$ and t-SNAREs ${ }^{14-16}$ in the split t-SNARE version of the fusion assay, we examined these interactions in detail in the following section.

Impact of linker mutations on syt•effector interactions. We assessed the t-SNARE-binding activity of WT and linker mutant forms of syt using a co-flotation assay. Each version of syt was mixed with proteoliposomes harbouring preformed t-SNARE heterodimers in the presence and absence of $\mathrm{Ca}^{2+}$; 1,2-dioleoyl-sn-glycero-3-[phospho-1-serine] (PS) was omitted to avoid interference by the PS-binding activity of $s y t^{13}$. Vesicles were isolated via density gradient centrifugation, subjected to SDS-PAGE, and proteins were visualized by staining with Coomassie blue (Supplementary Fig. 1a). Bound syt was quantified by densitometry (Supplementary Fig. 1b,c). Surprisingly, the t-SNARE-binding activities of all of the linker mutants were as good as, or better than, WT syt. Some degree of periodicity was observed, but this was not well fitted by a wave function with a period of three. Together, these results indicate the loss-of-function observed in the in vitro membrane fusion assay was not due to impaired binding of the linker mutants to t-SNAREs. We next examined the interaction of the mutants with anionic phospholipids.

Upon binding to membranes, the distal tips of the $\mathrm{Ca}^{2+}$-binding loops of syt partially insert into the hydrophobic 
core of the bilayer ${ }^{4,39}$. This property has been proposed to drive lipid rearrangements that accelerate fusion as outlined in the Introduction. Indeed, it was recently shown that the membrane penetration activity of syt does in fact constitute a critical step in excitation-secretion coupling ${ }^{4}$. We therefore subjected the linker mutants to in vitro membrane penetration assays by labelling the tip of $\mathrm{Ca}^{2+}$-binding loop 3 of C2A (F234C) or C2B (I367C) with $N$-(7-nitro-2,1,3-benzoxadiazol-4-yl) (NBD). Labelled protein was incubated with liposomes; upon addition of $\mathrm{Ca}^{2+}$, the probe in both $\mathrm{C} 2$-domains inserted into bilayers, as evidenced by the observed increase in emission intensity and the shift to shorter wavelengths (Fig. 3b,c) $)^{15}$. The fluorescence intensity changes were quantified and found to differ significantly among the linker mutants (Fig. 3d,e). As in the reconstituted fusion reactions, but in contrast to t-SNARE-binding assays, the
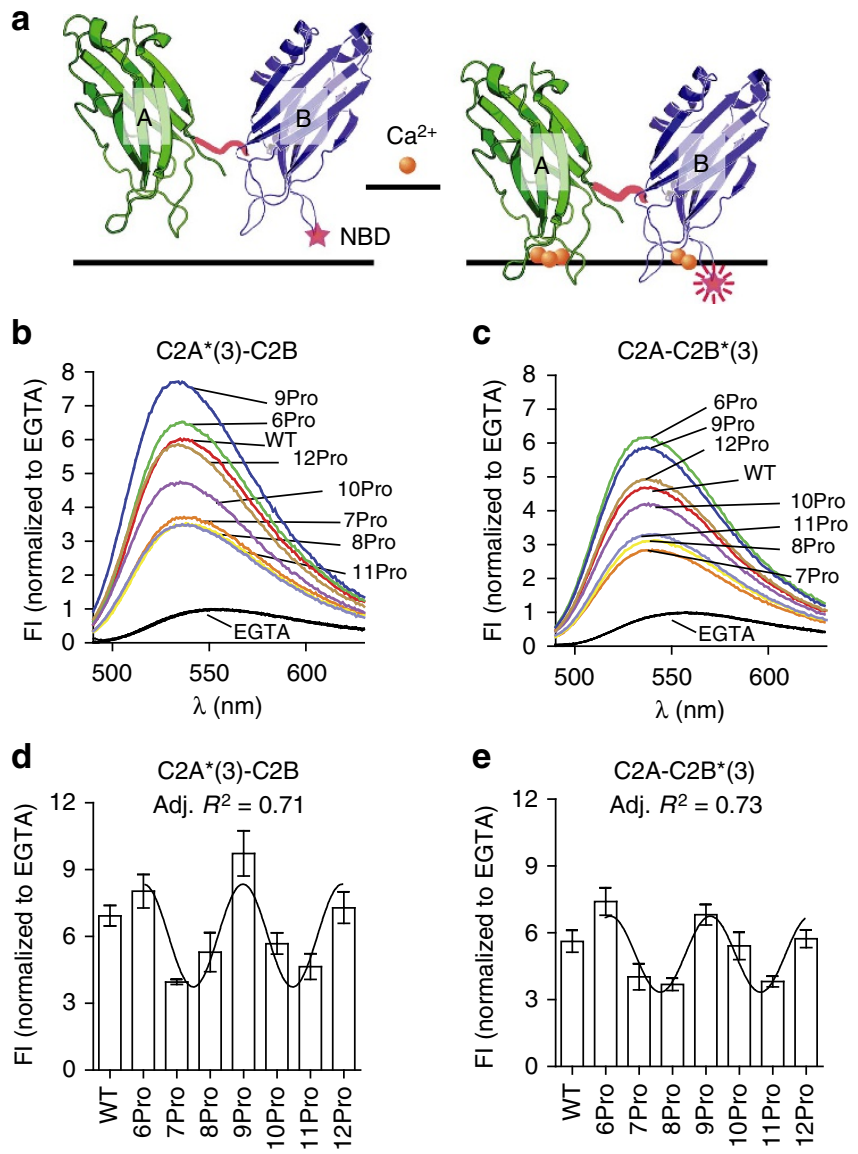

Figure 3 | Membrane penetration activity of WT and linker mutant forms of syt. (a) Schematic of the in vitro membrane penetration assay. The tip of loop 3 , in the $\mathrm{C} 2 \mathrm{~A}$ or $\mathrm{C} 2 \mathrm{~B}$ domain of syt, was labelled with NBD; the modified proteins are designated as $C 2 A^{*}(3)-C 2 B$ and $C 2 A-C 2 B^{*}(3)$, respectively ${ }^{15,39}$. The NBD probe exhibits an increase in fluorescence intensity (FI), and a blue shift in its emission spectrum, upon $\mathrm{Ca}^{2+}$. triggered penetration into lipid bilayers (harbor $15 \% \mathrm{PS})^{15,39}$. (b,c) Normalized NBD fluorescence spectra of $C 2 A^{\star}(3)-C 2 B$ or $C 2 A-C 2 B^{\star}(3)$ obtained in the absence ( $0.2 \mathrm{mM} \mathrm{EGTA}$; superimposed black traces) or presence $(1 \mathrm{mM})$ of $\mathrm{Ca}^{2+}$ (labelled grey traces). Representative spectra are shown; the protein concentration was $1 \mu \mathrm{M}$. (d,e) Averaged peak values of the NBD fluorescence of $C 2 A^{*}(3)-C 2 B$ or $C 2 A-C 2 B^{*}(3)$ in $1 \mathrm{mM} \mathrm{Ca}^{2+}$, normalized to the signals in EGTA. Data are represented as mean \pm s.e.m. For each condition, three independent trials were carried out. Data were fitted using a sine wave function with a periodicity of three; an adjusted (Adj.) $R^{2}$ value was generated to assess the goodness of the fit. membrane penetration activity of the linkers yielded a clear periodicity of three, and 6Pro, 9Pro and 12Pro exhibited the greatest activity. These findings were confirmed using a co-sedimentation assay to measure the apparent affinities of each linker mutant for membranes. As with the membrane penetration data, the affinity data also exhibited a periodicity of three (Supplementary Fig. 2). These results further demonstrate that the orientation of the tandem C2-domain of syt strongly influence the biochemical and biophysical properties of the protein. These results confirm the findings from the MD simulations, as a periodicity of three can only occur if the C2-domain constrained.

Linker mutants maintain the size of readily releasable pool. To determine the physiological consequences that result from altering the relative orientation of the $\mathrm{C} 2$-domains of syt in synapses, we used lentivirus to express full-length versions of each mutant in hippocampal neurons derived from syt knockout (KO) mice. All of the linker mutants were correctly targeted to presynaptic boutons, as revealed by their co-localization with synaptophysin (physin), a SV marker (Supplementary Fig. 3).

An important function of syt is to form or maintain the size of readily releasable pool (RRP) of SVs; in syt KO neurons, the RRP, measured via the application of hypertonic sucrose ${ }^{40}$, was significantly reduced ${ }^{4}$. Using the same approach, we observed that each of the poly-proline linker mutants was able to restore the RRP when expressed in syt KO neurons (Supplementary Fig. 4). These observations further show that all of the linker mutants are functional in synapses, and that alterations in the relative orientation of $\mathrm{C} 2 \mathrm{~A}$ and $\mathrm{C} 2 \mathrm{~B}$ have no effect on the ability of syt to regulate the RRP.

Linker mutants differentially regulate two modes of release. In the next series of experiments, we measured the ability of the linker mutants to rescue the loss of rapid evoked synaptic transmission in syt $\mathrm{KO}$ neurons. As expected, fast release was largely absent in syt KO neurons, but was fully rescued by WT syt (Fig. 4). All of the linker mutant forms of syt were able to restore the time latency between presynaptic stimulation and peak of the EPSCs (time-to-peak; Supplementary Table 1). However, the degree of rescue of the EPSC peak amplitude differed significantly among the mutants (Fig. 4b). Because the size of the RRP was identical among all conditions (Supplementary Fig. 4), differences in EPSC amplitude were due to differences in the ability of the linker mutants to trigger SV exocytosis and were not secondary to reductions in releasable vesicles. Importantly, the extent of fast neurotransmitter release in synapses exhibited a periodicity of three, identical to the periodicity observed for in vitro fusion assays and syt-membrane interactions. Briefly, 6Pro, 9Pro and 12Pro completely rescued the fast phase of neurotransmission, whereas the other constructs yielded partial recovery of evoked release (Fig. 4b). Together with the findings above, we conclude that the relative orientation between $\mathrm{C} 2 \mathrm{~A}$ and $\mathrm{C} 2 \mathrm{~B}$ is crucial for syt to function as a fast $\mathrm{Ca}^{2+}$ sensor during synaptic transmission, and suggest that syt drives fusion when its C2-domains point in the same direction.

We next examined the abilities of the linker mutants to clamp spontaneous release (that is, miniature excitatory postsynaptic currents; mEPSC). mEPSCs were recorded from syt KO neurons expressing 6Pro, 7Pro, 8Pro and 9Pro mutants of syt, which cover a complete period of the relative angle between C2-domains. Strikingly, a periodicity of three was also found for the frequency of mEPSC events. As shown in Fig. 5a,b, syt $\mathrm{KO}$ neurons expressing 6Pro and 9Pro exhibited no differences in mEPSC frequency as compared with control syt $\mathrm{KO}$ neurons, whereas 
a
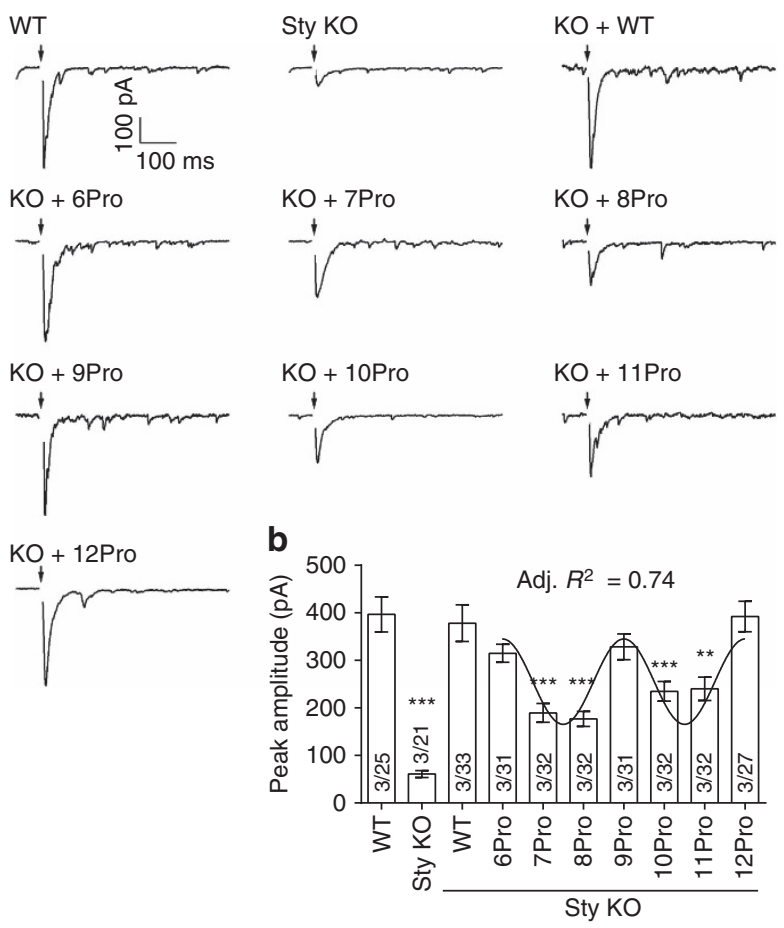

Figure 4 | The ability of linker mutants to rescue evoked synaptic transmission exhibits periodicity. (a) Representative evoked EPSCs recorded from $\mathrm{WT}$, syt $\mathrm{KO}$ and syt $\mathrm{KO}$ neurons expressing $\mathrm{WT}$ or proline linker mutant forms of syt. Black arrows indicate depolarization. (b) Peak amplitudes of evoked EPSCs are plotted. The 6Pro, 9Pro and 12Pro mutants fully rescued the peak amplitude; all other mutants yielded only partial rescue. The degree of rescue was well fitted by a sine wave function with a periodicity of three; an adjusted (Adj.) $R^{2}$ value was generated to assess the goodness of the fit. Mean values \pm s.e.m. are plotted. For each condition, data were collected from 21 to 33 cells from a total of six coverslips, where two coverslips were obtained from each of three independent litters of mice. Recording were made from 3 to 7 cells per coverslip. ${ }^{\star \star} P<0.001$, ${ }^{* \star *} P<0.001$ versus $W T$, one-way analysis of variance (ANOVA) followed by Tukey's multiple comparisons test. The number of independent litters, $N$, and the number of cells, $n$, are indicated in the bar graph as $N / n$. Full results of the one-way ANOVA are in Supplementary Table 4.

7Pro and 8Pro clamped spontaneous synaptic transmission to a same extent as WT syt. The amplitude of the mEPSCs were the same for WT and all of the mutants (Fig. 5c).

Structural states of syt that subserve distinct functions. The evoked and spontaneous release data for the 6-9Pro linker mutants were normalized relative to WT, and plotted in Fig. $6 \mathrm{c}$, revealing a reciprocal relationship between the mutants that drove evoked release and the mutants that inhibited spontaneous release. Linker mutants that drive efficient evoked release are less effective at clamping spontaneous release, whereas mutants that effectively clamp spontaneous release fail to drive efficient evoked transmission. These results demonstrate that syt can switch between at least two different functional states via changes in the relative orientation of its tandem $\mathrm{C} 2$-domains.

In the final series of experiments, we used $\mathrm{PET}^{42}$ to experimentally address the relative orientations of the tandem C2-domains in WT and the 6-9Pro linker mutants. We labelled the same positions used for the membrane penetration assays above (that is, membrane penetration loop 3 in both $\mathrm{C} 2 \mathrm{~A}$ and $\mathrm{C} 2 \mathrm{~B})$. However, in this case, the engineered Cys residue in $\mathrm{C} 2 \mathrm{~A}$ a

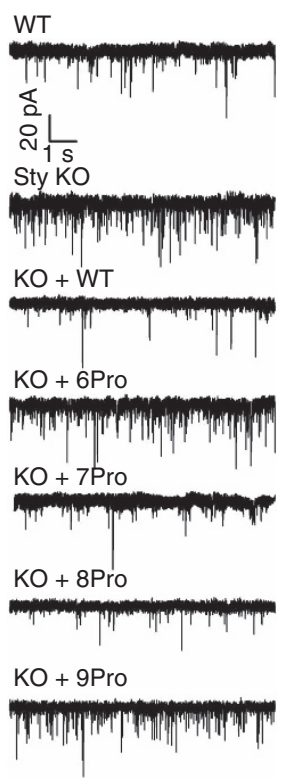

b

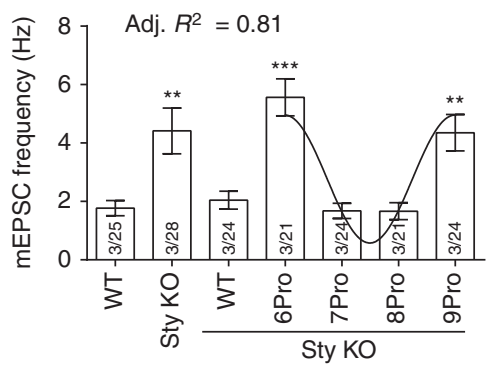

C

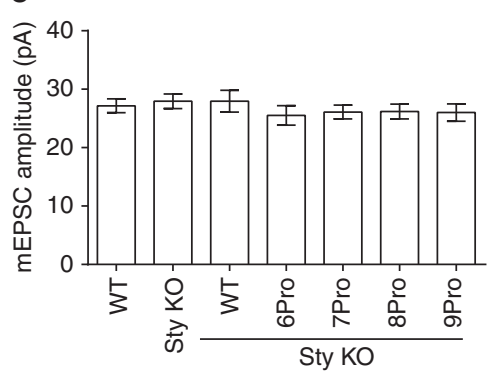

Figure 5 | Linker mutants yield differences in spontaneous release rates. (a) Representative mEPSC traces recorded from WT, syt KO and syt KO neurons expressing WT or proline linker mutant forms of syt. The frequency (b) and amplitude (c) of mEPSCs are plotted as mean value \pm s.e.m. The 6 Pro and 9Pro mutants were unable to clamp spontaneous fusion, whereas 7 Pro and 8 Pro had robust clamping activity. For each condition, data were collected from 21 to 28 cells from a total of six coverslips, where two coverslips were obtained from each of three independent litters of mice. Recording were made from 3 to 7 cells per coverslip. ${ }^{\star \star} P<0.001$,

${ }^{\star * \star} P<0.001$ versus WT, one-way analysis of variance (ANOVA) followed by Tukey's multiple comparisons test. The number of independent litters, $N$, and the number of cells, $n$, are indicated in the bar graph as $N / n$. One-way ANOVA results are provided in Supplementary Tables 5 and 6.

was modified with the boron-dipyrromethene dye BODIPY FL (Bpy); the PET quencher, Trp, was placed in C2B (Fig. 6a). The PET quenching efficiency for the 6-9Pro linker mutant constructs were determined and the results revealed a periodicity of three (Fig. 6b). Maximal quenching was observed for 6 and 9Pro, whereas the 7 and 8Pro yielded little quenching. As Trp-mediated PET quenching occurs on the $0.5-1.5 \mathrm{~nm}$ distance scale, these results indicate that in the 6 and 9Pro mutants, the tandem $\mathrm{C} 2$-domains are in the closest proximity, and hence point in the same direction, whereas 7 and 8Pro are further apart so the $\mathrm{C} 2$-domains point in different directions.

We then normalized the PET quenching data, and overlaid these results onto the evoked and spontaneous release plots shown in Fig. 6c. From this comparison, it is apparent that syt drives evoked release when its tandem $\mathrm{C} 2$-domains point in the same direction (for example, 6Pro, 9Pro, and by inference, 12Pro), and syt clamps fusion when the C2-domains point in different directions.

\section{Discussion}

Because the individual C2-domains of syt do not appear to undergo large conformational changes 8,9 , we propose that changes in the relative disposition of the $\mathrm{C} 2$-domains underlie the ability of syt to switch between functional states. In this model, large conformation changes in syt can be achieved without significant structural changes within the individual domains. Previous X-ray crystallography studies shed some light on this 
a

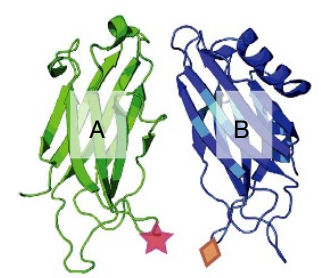

High quenching

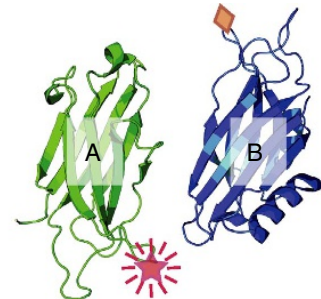

Low quenching b

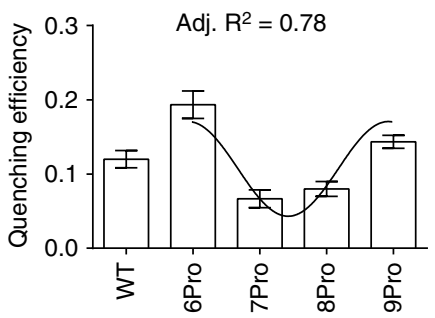

C

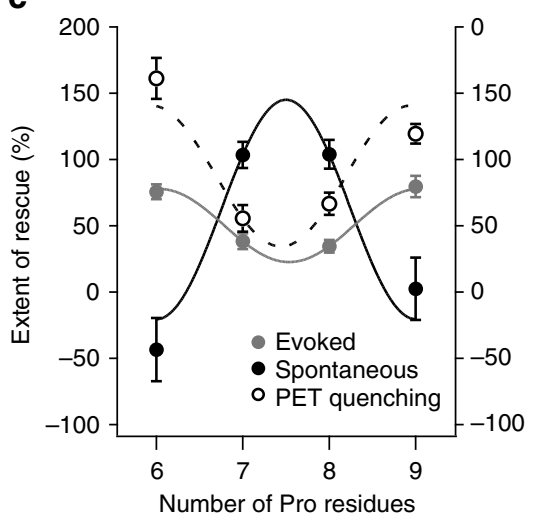

d

Resting Activated
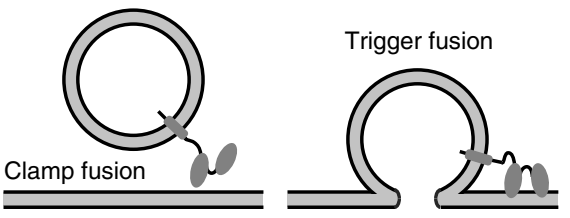

Figure 6 | Distinct relative C2-domain orientations underlie syt regulation of evoked and spontaneous release. (a) IIlustration showing the PET quenching assay, showing the quenching Trp (diamond) and the BODIPY fluorophore (star) that is quenched, in the membrane penetration loops of C2A and $\mathrm{C} 2 \mathrm{~B}$, respectively. (b) The quenching efficiencies were calculated and plotted for the WT and 6-9Pro mutants; $0.5 \mu \mathrm{M}$ protein was used in each condition. Data were fitted using a sine wave function with a periodicity of three; an adjusted (Adj.) $R^{2}$ value was generated to assess the goodness of the fit. The most efficient quenching was observed for 6Pro and 9Pro, so the C2-domains in these two constructs point in the same direction; for 7Pro and 8Pro, the C2-domains are not in a parallel relative orientation. Data are represented as mean \pm s.e.m.; for each condition, three independent trials were carried out. (c) Reciprocal abilities of the syt linker mutants to clamp spontaneous release and to drive evoked synaptic transmission. The amplitude of evoked EPSCs and the frequency of mEPSC were used to evaluate the function each linker mutant. Data were normalized using values obtained from WT (100\%) and syt KO neurons ( $0 \%)$. Again, the results were fitted with a sine wave function with a periodicity of three; an adjusted $R^{2}$ value was generated to assess the goodness of the fit. For completeness, the PET quenching data were normalized and overlaid onto this plot, to reveal the relative orientations that underlie the regulation of evoked versus spontaneous release. (d) Under resting conditions, C2A and C2B point to different directions to clamp fusion; when activated by $\mathrm{Ca}^{2+}, \mathrm{C} 2 \mathrm{~A}$ and $\mathrm{C} 2 \mathrm{~B}$ switch to a parallel configuration to trigger SV exocytosis.

question. In one structure of syt, the $\mathrm{Ca}^{2+}$-binding loops of $\mathrm{C} 2 \mathrm{~A}$ and $\mathrm{C} 2 \mathrm{~B}$ were roughly perpendicular to one-another ${ }^{31}$, suggesting that one $\mathrm{C} 2$-domain might penetrate the plasma membrane while the other C2-domain could penetrate a fusion neck or stalk ${ }^{43}$. However, in another crystal structure, in this case of the cytoplasmic domain of syt3, the tandem $\mathrm{C} 2$-domains formed a shallow v-shape, with the $\mathrm{Ca}^{2+}$-binding loops of $\mathrm{C} 2 \mathrm{~A}$ and $\mathrm{C} 2 \mathrm{~B}$ largely pointing towards one-another ${ }^{29}$. In contrast, two other groups proposed a model in which $\mathrm{C} 2 \mathrm{~A}$ and $\mathrm{C} 2 \mathrm{~B}$ have an 'antiparallel conformation' such that the $\mathrm{Ca}^{2+}$-binding loops of $\mathrm{C} 2 \mathrm{~A}$ and $\mathrm{C} 2 \mathrm{~B}$ point in opposite directions ${ }^{27,44}$. In another study, based on nuclear magnetic resonance measurements, it was concluded that the relative orientation is dynamic, but that there might be a limited set of preferred states ${ }^{30}$. In a newer nuclear magnetic resonance study, it was demonstrated that the 'parallel orientation' of the C2-domains of syt is the most favourable conformation when engaging membranes (nanodiscs) in the presence of $\mathrm{Ca}^{2+}$ (ref. 45). Finally, kinetics studies showed that, in response to $\mathrm{Ca}^{2+}, \mathrm{C} 2 \mathrm{~A}$ and $\mathrm{C} 2 \mathrm{~B}$ simultaneously penetrate the target membrane, suggesting that the tandem $\mathrm{C} 2$-domains point in the same direction ${ }^{24,39}$. Given the distinct views concerning the relative disposition of the tandem C2-domains of syt, we addressed the debate by replacing the nine-residue linker connecting the tandem C2-domains of syt with a series of poly-proline linkers, which constrain the relative orientation of $\mathrm{C} 2 \mathrm{~A}$ and $\mathrm{C} 2 \mathrm{~B}$ to specific, stable, states. We then carried out biochemical and cell-based experiments to discern the functionality of each of these constrained forms of the protein.

Strikingly, adding or removing single residues from the nine-residue poly-proline rod revealed a clear periodicity of three in all but one of the assays that were carried out; the lone exception was the t-SNARE-binding activity of the linker mutants. The periodicity was evident in in vitro reconstituted fusion assays, membrane penetration/binding assays, PET quenching experiments and electrophysiological measurements of evoked and spontaneous release. For example, among all the linker mutants, the 6Pro, 9Pro, 12Pro fully rescued evoked synaptic transmission, whereas the other linker mutants yielded impaired EPSCs. The MD simulations, in conjunction with our experimental data, indicate that in 6Pro, 9Pro and 12Pro, the tandem C2-domains of syt point the same direction; syt drives efficient SV exocytosis when its tandem C2-domains are in a parallel configuration.

We compared evoked with spontaneous release regulated by each of the linker mutants, and a clear reciprocal relationship emerged (Fig. 6c). These results help to explain how syt is able to regulate distinct forms of exocytosis: at rest, the C2-domains point in different directions to inhibit spontaneous SV exocytosis. Then, in response to $\mathrm{Ca}^{2+}$, syt changes states via large-scale alterations in the disposition of its tandem C2-domains; these relative movements allow both domains point in the same direction to interact with the plasma membrane to drive fusion (Fig. 6d) $)^{24,39,46}$. 
There are a variety of proteins that contain tandem C2-domains, and myriad other structural motifs. The rigidity of poly-proline rods can be further exploited to explore the functional interactions between these domains, and to alter their relative orientations, as described here.

\begin{abstract}
Methods
Molecular biology. cDNA encoding rat syt ${ }^{47}$ was provided by T.C. Südhof (Stanford University); the D374 mutation was corrected by replacement with a glycine residue ${ }^{48}$. The indicated poly-proline linker mutant forms of syt were generated via PCR using the overlapping primer method ${ }^{49}$. Full-length versions were subcloned into a modified pLOX vector (Addgene) ${ }^{50}$; fragments encoding the cytoplasmic domains were subcloned into pGEX-4T vectors. For site-directed labelling, the lone native cysteine (Cys277) in the cytoplasmic domain of syt was substituted with an alanine. Then, a single cysteine was introduced at Phe234 of C2A, or Ile367 of C2B. Point mutations were generated using a QuikChange Site-Directed Mutagenesis Kit (Stratagene). WT and linker mutant forms of the cytoplasmic domain of syt (residues 96-421) were expressed as glutathione $S$-transferase fusion proteins, purified using glutathione-Sepharose beads (GE healthcare), and cleaved with thrombin to remove glutathione $S$-transferase ${ }^{23}$. cDNA encoding full-length rat syntaxin-1A, mouse SNAP-25B and mouse synaptobrevin-2 was provided by R.H. Scheller (Genentech), M.C. Wilson (Univ. New Mexico) and J.E. Rothman (Yale University), respectively. Each SNARE cDNA was subcloned into a pTrcHis vector (Invitrogen), resulting in an $\mathrm{N}$-terminal $\mathrm{His}_{6}$-tag that was used for purification ${ }^{14}$. The original sequences of all primers used in this study are provided in Supplementary Table 2.
\end{abstract}

Hippocampal neuronal culture and viral infection. Syt KO mice were obtained from Jackson Laboratory, and a colony was established. Primary cultures of hippocampal neurons were prepared from syt KO mice, or their WT littermates, at postnatal day 0 , in accordance with the guidelines of the National Institutes of Health, as approved by the Animal Care and Use Committee at the University of Wisconsin. Briefly, hippocampi were isolated from WT and KO mice, washed with Hank's buffered salt solution and digested for $30 \mathrm{~min}$ in the same buffer plus $0.25 \%$ (w/v) trypsin and $2.21 \mathrm{mM} \mathrm{EDTA}$ at $37^{\circ} \mathrm{C}$. Hippocampi were transferred to Dulbecco's Modified Eagle Medium supplemented with $10 \%$ fetal bovine serum (Hyclone), and then dissociated mechanically. The dissociated neurons were plated on $12 \mathrm{~mm}$ glass coverslips (Warner Instruments), coated with poly-D-lysine, at a density of $25,000-40,000$ cells per $\mathrm{cm}^{2}$. Neuronal cultures were maintained in Neurobasal-A medium, supplemented with B27 and GlutaMAX (Invitrogen), at $37^{\circ} \mathrm{C}$ in a $5 \% \mathrm{CO}_{2}$ humidified incubator.

To generate viruses, the syt pLOX constructs were co-transfected into HEK-293T cells (American Type Culture Collection, not authenticated, not tested for micoplasma) with two lentiviral packaging vectors (vesicular stomatitis virus $G$ glycoprotein and Delta 8.9 $)^{50}$. Three days after transfection, lentivirus was harvested from the media by centrifugation at $126,000 \mathrm{~g}$ for $2 \mathrm{~h}$. Neuronal cultures were infected with virus at 5 DIV (days in vitro). The infection rate was $\sim 90 \%$, as estimated by the co-expression of GFP.

Immunocytochemistry. At 13-15 DIV, neuronal cultures were fixed with $4 \%$ paraformaldehyde $(\mathrm{w} / \mathrm{v})$ in PBS for $15 \mathrm{~min}$, permeabilized in $0.1 \%$ Triton X-100 (v/v) for $10 \mathrm{~min}$ and then blocked in $10 \%$ bovine serum albumin (w/v) plus $0.1 \%$ Triton X-100 (v/v) for $30 \mathrm{~min}$. Coverslips were incubated with a mouse monoclonal antibody against syt (mAb48, diluted 1:1,000, Developmental Studies Hybridoma Bank $)^{51}$ and, to visualize nerve terminals, a polyclonal guinea pig antibody against physin (Cat. No. 101004, diluted 1:1,000; Synaptic System) for 2 h. Coverslips were washed three times with PBS and incubated with Alexa 488-tagged anti-mouse and Alexa 594-tagged anti-guinea pig secondary antibodies (Cat. No. 115-547-187 and 706-586-148; diluted 1:400; Jackson ImmunoResearch Laboratories) for $1 \mathrm{~h}$. Coverslips were again washed three times and mounted in Fluoromount (Southern Biotechnology Associates). Images were acquired using an Olympus FV1000 confocal microscope with a $\times 601.40 \mathrm{NA}$ oil objective, using fixed laser intensity and gain settings. To quantify the co-localization of each of the syt constructs with physin, the Mander's coefficients (syt to physin) for each image pair were calculated using the JACoP plug-in (ref. 52) in ImageJ software.

\section{Electrophysiology. Whole-cell patch clamp recordings were obtained from} cultured hippocampal neurons at 13-17 DIV. During recordings, neurons were continuously perfused with bath solution $(128 \mathrm{mM} \mathrm{NaCl}, 30 \mathrm{mM}$ glucose, $5 \mathrm{mM}$ $\mathrm{KCl}, 2 \mathrm{mM} \mathrm{CaCl} 2,1 \mathrm{mM} \mathrm{MgCl} 2,50 \mu \mathrm{M}$-AP5, $20 \mu \mathrm{M}$ bicuculline and $25 \mathrm{mM}$ HEPES, pH 7.3). The recording pipettes were pulled to a resistance of 3-5 M $\Omega$ from glass capillaries (Warner Instruments) and filled with standard intracellular solution (130 mM K-gluconate, $1 \mathrm{mM}$ EGTA, $5 \mathrm{mM}$ Na-phosphocreatine, $2 \mathrm{mM}$ Mg-ATP, $0.3 \mathrm{mM}$ Na-GTP, $5 \mathrm{mM}$ QX-314 and $10 \mathrm{mM}$ HEPES, $\mathrm{pH}$ 7.3). Neurons were voltage clamped at $-70 \mathrm{mV}$ via the recording pipettes using a MultiClamp $700 \mathrm{~B}$ amplifier (Molecular Devices). Only whole-cell patches with series resistances $<15 \mathrm{M} \Omega$ were used for recording.
For evoked EPSCs, the presynaptic neuron was depolarized by a 1-ms voltage step $(40 \mathrm{~V})$ using a bipolar electrode pulled from theta glass capillary (Warner Instruments) and filled with bath solution. For the RRP measurement, the field of view (at $\times 40)$ around the patch-clamped neuron was locally perfused with bath solution plus $500 \mathrm{mM}$ sucrose for $10 \mathrm{~s}$ using a Picospritzer III (Parker), releasing the entire RRP of all connected presynaptic boutons. For mEPSCs, $1 \mu \mathrm{M}$ tetrodotoxin was present in the bath solution. All experiments were performed at room temperature (RT). Data were acquired at a sampling rate of $10 \mathrm{kHz}$ and filtered at $2.8 \mathrm{kHz}$ using the pClamp (Molecular Devices) software. Off-line analysis was performed using the Clampfit (Molecular Devices) and Igor (WaveMetrics) software. The D-AP5, bicuculline, tetrodotoxin and QX-314 were purchased from TOCRIS Bioscience. All recordings were obtained from randomly selected cells.

Liposomes. Synthetic 1, 2-dioleoyl-sn-glycero-3-phospho-l-serine (phosphatidylserine, PS), 1-palmitoyl-2-oleoyl-sn-glycero-3-phosphoethanolamine (phosphatidylethanolamine, PE), 1-palmitoyl-2-oleoyl-sn-glycero-3-phosphocholine (phosphatidylcholine, PC), 1,2-dipalmitoyl-sn-glycero-3-phospho-ethanolamine$\mathrm{N}$-(7-nitro-2-1, 3-benzoxadiazol-4-yl) (NBD-PE) and N-(lissamine rhodamine B sulfonyl)-1, 2-dipalmitoyl-sn-glycero-3-phosphoethanolamine (rhodamine-PE) were obtained from Avanti Polar Lipids. Protein-free SUVs (small unilamellar vesicles) were prepared as follows: lipids (15\% PS $+30 \%$ PE $+55 \%$ PC or $25 \%$ $\mathrm{PS}+30 \% \mathrm{PE}+45 \% \mathrm{PC}$, as indicated) were mixed and dried under nitrogen, and lyophilized for $1 \mathrm{~h}$. The dried lipid film was resuspended in Tris buffer $(50 \mathrm{mM}$ Tris, $\mathrm{pH} 7.4,150 \mathrm{mM} \mathrm{KCl})$ and extruded 20 times through $100 \mathrm{~nm}$ polycarbonate filters. SNARE-bearing SUVs were prepared ${ }^{14}$ using $15 \% \mathrm{PS}+30 \%$ $\mathrm{PE}+55 \% \mathrm{PC}$ for $\mathrm{t}$-SNARE vesicles (Tr) and $15 \% \mathrm{PS}+27 \% \mathrm{PE}+55 \% \mathrm{PC}+1.5 \%$ $\mathrm{NBD}-\mathrm{PE}+1.5 \%$ rhodamine-PE for $\mathrm{v}$-SNARE vesicles $(\mathrm{Vr})$.

Labeling of the cytoplasmic domain of syt with NBD. For labelling syt with NBD for membrane penetration experiments, the appropriate purified cysteine mutants were adjusted to $\sim 10 \mu \mathrm{M}$ in Tris buffer ( $50 \mathrm{mM}$ Tris, $\mathrm{pH} 7.4,150 \mathrm{mM}$ $\mathrm{KCl}$ ). NBD (Molecular Probes) was then added drop-wise to each protein solution to reach a final concentration of $\sim 150 \mu \mathrm{M}$; samples were incubated at RT for $2 \mathrm{~h}$ with rotation, and free dye was removed by extensive dialysis against Tris buffer ( $50 \mathrm{mM}$ Tris, $\mathrm{pH} 7.4,150 \mathrm{mM} \mathrm{KCl}$ ). The NBD concentration was determined by absorbance using a molar extinction coefficient of $25,000 \mathrm{M}^{-1} \mathrm{~cm}^{-1}$ at $480 \mathrm{~nm}$. The protein concentration was determined by SDS-PAGE and staining with Coomassie blue, using BSA as a standard. The labelling stoichiometry was $0.7-0.9 \mathrm{~mol}$ of dye per mole of protein.

Fluorescence measurements. Membrane penetration assays were performed at RT using a PTI QM-1 fluorometer and FELIX software (Photon Technology International). For membrane penetration assays, NBD-labelled protein was mixed with SUVs (113 $\mu \mathrm{M}$ total lipid) in Tris buffer $(50 \mathrm{mM}$ Tris, $\mathrm{pH} 7.4,150 \mathrm{mM} \mathrm{KCl})$ in the presence of either EGTA ( $0.2 \mathrm{mM}$ final concentration) or $\mathrm{Ca}^{2+}(1 \mathrm{mM}$ final free concentrations). NBD was excited at $470 \mathrm{~nm}$, and the emission spectra (490 to $630 \mathrm{~nm}$; 4-nm slits) were corrected for blank and instrument response.

Co-sedimentation assays. WT or linker mutant cytoplasmic domains of syt $(4 \mu \mathrm{M})$ were mixed with PS-bearing liposomes (at the indicated concentrations) in Tris buffer $(50 \mathrm{mM}$ Tris, pH 7.4, $150 \mathrm{mM} \mathrm{KCl})$, in the presence of $1 \mathrm{mM} \mathrm{Ca}^{2+}$ or $0.2 \mathrm{mM}$ EGTA, and then centrifuged at $170,000 \mathrm{~g}$ at $4{ }^{\circ} \mathrm{C}$ for $45 \mathrm{~min}$ in a Beckman Optima MAX-E (Beckman Coulter) tabletop ultracentrifuge. The supernatant from each sample was collected and analysed by SDS-PAGE and Coomassie blue staining. Bands were quantified by densitometry to reveal the amount of free protein, and these data were used to calculate the amount of bound protein, which was then plotted versus the PS concentration $(\mathrm{mM})$; apparent $K_{\mathrm{d}}$ values were determined by fitting the data with 'one-site-specific binding curves' using Prism 6 (Graphpad Software). An uncropped image of entire gel is provided in Supplementary Fig. 5.

Co-flotation assays. One hundred-microlitre reactions contained $11 \mu \mathrm{M}$ WT or linker mutant forms of the cytoplasmic domain of syt, $45 \mu \mathrm{l}$ of PS-free t-SNARE vesicles and reconstitution buffer (25 mM HEPES-KOH, pH 7.4, $100 \mathrm{mM} \mathrm{KCl}, 10 \%$ glycerol, $1 \mathrm{mM}$ dithiothreitol) ${ }^{13}$. Samples contained either $0.2 \mathrm{mM}$ EGTA or $1 \mathrm{mM}$ free $\mathrm{Ca}^{2+}$, and were incubated at RT for 45 min with shaking, loaded onto an Accudenz gradient, centrifuged at $287,000 \mathrm{~g}$, and the vesicles were collected at the $0-30 \%$ Accudenz interface. Samples were then subjected to SDS-PAGE and stained with Coomassie blue; protein bands were quantified by densitometry. Uncropped images of entire gels are provided in Supplementary Fig. 5.

In vitro fusion assays. 'Split t-SNARE' fusion assays were carried out ${ }^{15}$; in this variant of the fusion assay, syx is reconstituted but SNAP-25 is added in a soluble form. This configuration of the assay bypasses the ability of syt to stimulate fusion by merely aggregating $\mathrm{v}$ - and t-SNARE vesicles when the t-SNAREs, syx and SNAP-25, are reconstituted as pre-formed heterodimers. Rather, $\mathrm{Ca}^{2+} \bullet$ syt must direct the folding of SNAP-25 onto syx, resulting in the assembly of functional trans SNARE complexes ${ }^{14}$, thus driving fusion. Samples were incubated at $37^{\circ} \mathrm{C}$ 
for $30 \mathrm{~min}$ in the presence of $0.2 \mathrm{mM}$ EGTA, followed by addition of $\mathrm{Ca}^{2+}$ ( $1 \mathrm{mM}$ final free concentration). Fusion was monitored, as dequenching of NBD fluorescence, for an additional $1 \mathrm{~h}$. The detergent, $\beta$-D-maltoside $(0.5 \% \mathrm{w} / \mathrm{v})$, wa then added, resulting in 'infinite' dilution of the NBD-rhodamine fluorescence resonance energy transfer pair, yielding the maximum fluorescence signal used to normalize the data.

PET quenching assay. A single Cys was placed at position F234C in the cytoplasmic domain of syt as described above. A second version was constructed, in which the F234C mutant also included a second mutation, I367W. The Cys residue, in both constructs, was labelled using a fivefold molar excess of the maleimide derivative of BODIPY FL (Life Technologies), for $2 \mathrm{~h}$ at $25^{\circ} \mathrm{C}$ in labelling buffer (20 mM HEPES, pH 7.4, $100 \mathrm{mM} \mathrm{KCl,} 10 \%$ glycerol). The reaction was quenched by addition of $5 \mathrm{mM}$ dithiothreitol, and free dye was removed by gel filtration on a Superdex $20010 / 300$ column (GE Healthcare) in labelling buffer. The labelling efficiency was $\sim 80 \%$ for both constructs. PET measurements, using the Trp in C2B to quench the fluorescence of the BODIPY in C2A, were carried out $^{42}$. Briefly, fluorescence measurements were performed in reconstitution buffer at $25^{\circ} \mathrm{C}$; samples were excited at $470 \mathrm{~nm}$, and the emission was collected from 500 to $600 \mathrm{~nm}$. The efficiency of quenching was calculated by comparing the BODIPY fluorescence intensity in the absence and presence of the Trp quencher.

\section{Simulations and free energy computations with metadynamics. All MD} simulations were performed using NAMD 2.9 (ref. 53) and the CHARMM22 force field ${ }^{54,55}$ for proteins. Simulations were carried out in the constant pressure-temperature ensemble at $1 \mathrm{~atm}$ and $298 \mathrm{~K}$ using Nosé-Hoover and Langevin piston pressure coupling protocols. Particle Mesh Ewald was used for the electrostatics and Lennard-Jones interactions were treated with a cutoff of $12 \AA$ and a switching function turned on at $10 \AA$.

To characterize the rotational flexibility of the poly-proline linker with respect to the $\mathrm{C} 2$-domains, we performed two-dimensional metadynamics simulations for individual C2-domains connected to a nine-proline linker (that is, C2A-9Pro and C2B-9Pro $)^{56}$ to compute the free energy map as a function of the two dihedral angles defined in Supplementary Fig. 6. These angles were chosen based on previous studies that characterize conformational isomerization of proline $\mathrm{e}^{57}$, and different dihedral angles were used for C2A-9Pro and C2B-9Pro because the 9Pro peptide is connected to $\mathrm{C} 2 \mathrm{~A}$ at its $\mathrm{N}$-terminus, whereas the linker is connected to $\mathrm{C} 2 \mathrm{~B}$ at its C-terminus. The built-in version of metadynamics in NAMD 2.9 was used. The Gaussian width was set to $9.0^{\circ}$ and the Gaussian height was $0.07 \mathrm{kcal} \mathrm{mol}^{-1}$; Gaussian was added every $0.2 \mathrm{ps}$. Convergence of the metadynamics simulation was monitored based on the distribution of the collective variables. The reported free energy maps in Supplementary Fig. 7 were obtained with $70 \mathrm{~ns}$ of metadynamics for each C2-domain.

For the starting structure, the coordinates for isolated $\mathrm{C} 2 \mathrm{~A}$ or $\mathrm{C} 2 \mathrm{~B}$ were extracted from PDB entry 2R83 (ref. 31); $\mathrm{Ca}^{2+}$ ions were not included in the model. The native linker, SAEKEEQEK, was replaced with a poly-proline peptide using CHARMM c37al (ref. 58). The system was then solvated with TIP3P water and $0.15 \mathrm{M}$ of $\mathrm{NaCl}$; the average box size during the simulation was $80 \times 80 \times 80 \AA^{3}$.

Following the metadynamics simulations for the individual domains, stable conformers were identified based on the computed free energy maps, and these were used to build models for $\mathrm{C} 2 \mathrm{~A}-\mathrm{C} 2 \mathrm{~B}$ connected with a poly-proline linker (C2A- $n$ Pro-C2B, where $n$ is the number of proline residues); three linker lengths ( $n=9,10$ and 11 ) were simulated. C2A- $n$ Pro-C2B were solvated with TIP3P water and $0.15 \mathrm{M}$ of $\mathrm{NaCl}$; the average box size during the simulation was $110 \times 110 \times 110 \AA^{3}$. The systems were simulated for at least $70 \mathrm{~ns}$. The relative orientations of the $\mathrm{Ca}^{2+}$-binding loops relative to poly-proline linker in the $\mathrm{C} 2$ domains were monitored and shown in Supplementary Fig. 8.

Statistical analysis. The sine wave traces, superimposed on the bar graph in Figs $2-6$, were generated by fitting the data using sine wave functions, with a periodicity of three, using Origin 9 (OriginLab), as follows:

$$
y=a+b \times \sin \left(\frac{x-c}{3} \times 2 \pi\right)
$$

in which $y$ corresponds to the measured variable, $x$ is the number of proline residues in the poly-proline linker and $a, b$ and $c$ are the offset, amplitude and phase parameters, respectively. The detailed results are listed in Supplementary Table 3.

For the in vitro biochemical studies, three independent trials were carried out. No further statistical analysis, besides fitting with the sine wave function, was carried out.

In all the electrophysiological experiments, the number of recorded cells, coverslips and independent litters of mice are detailed in the Figure Legends. Oneway analysis of variance followed by Tukey's multiple comparisons test were carried out to compare differences among groups. Detailed results of all statistical analysis are provided in the Supplementary Tables $4-7$. We did not perform any statistical tests to justify the sample sizes; however, the sample sizes in this study follow the standard used in this field ${ }^{4,41,59}$. We did not asses the normality of the results; however, we present the electro-physiological data as scatter plots so the variance of the raw data is evident.

Experiments were not carried out blind.

\section{References}

1. Stevens, C. F. Presynaptic function. Curr. Opin. Neurobiol. 14, 341-345 (2004)

2. Kavalali, E. T. et al. Spontaneous Neurotransmission: An Independent Pathway for Neuronal Signaling? Physiology 26, 45-53 (2011).

3. Sutton, M. A. et al. Miniature neurotransmission stabilizes synaptic function via tonic suppression of local dendritic protein synthesis. Cell 125, 785-799 (2006).

4. Liu, H. et al. Linker mutations reveal the complexity of synaptotagmin 1 action during synaptic transmission. Nat. Neurosci. 17, 670-677 (2014).

5. Chapman, E. R. How does synaptotagmin trigger neurotransmitter release? Annu. Rev. Biochem. 77, 615-641 (2008)

6. Littleton, J. T., Stern, M., Perin, M. \& Bellen, H. J. Calcium dependence of neurotransmitter release and rate of spontaneous vesicle fusions are altered in Drosophila synaptotagmin mutants. Proc. Natl Acad. Sci. USA 91, 10888-10892 (1994).

7. Littleton, J. T., Stern, M., Schulze, K., Perin, M. \& Bellen, H. J. Mutational analysis of Drosophila synaptotagmin demonstrates its essential role in $\mathrm{Ca}^{2+}$. activated neurotransmitter release. Cell 74, 1125-1134 (1993).

8. Shao, X. G., Fernandez, I., Sudhof, T. C. \& Rizo, J. Solution structures of the $\mathrm{Ca}^{2+}$-free and $\mathrm{Ca}^{2+}$-bound $\mathrm{C}(2) \mathrm{A}$ domain of synaptotagmin I: Does $\mathrm{Ca}^{2+}$ induce a conformational change? Biochemistry 37, 16106-16115 (1998).

9. Fernandez, I. et al. Three-dimensional structure of the synaptotagmin $1 \mathrm{C} 2 \mathrm{~B}$ domain: synaptotagmin 1 as a phospholipid binding machine. Neuron 32, 1057-1069 (2001).

10. Sollner, T., Bennett, M. K., Whiteheart, S. W., Scheller, R. H. \& Rothman, J. E. A protein assembly-disassembly pathway in-vitro that may correspond to sequential steps of synaptic vesicle docking, activation, and fusion. Cell 75, 409-418 (1993).

11. Jahn, R. \& Scheller, R. H. SNAREs--engines for membrane fusion. Nat. Rev. Mol. Cell Biol. 7, 631-643 (2006).

12. Weber, T. et al. SNAREpins: minimal machinery for membrane fusion. Cell 92, 759-772 (1998).

13. Tucker, W. C., Weber, T. \& Chapman, E. R. Reconstitution of $\mathrm{Ca}^{2+}$-regulated membrane fusion by synaptotagmin and SNAREs. Science 304, 435-438 (2004).

14. Bhalla, A., Chicka, M. C., Tucker, W. C. \& Chapman, E. R. Ca ${ }^{2+}$. synaptotagmin directly regulates t-SNARE function during reconstituted membrane fusion. Nat. Struct. Mol. Biol. 13, 323-330 (2006).

15. Hui, E. et al. Mechanism and function of synaptotagmin-mediated membrane apposition. Nat. Struct. Mol. Biol. 18, 813-821 (2011).

16. Yao, J., Gaffaney, J. D., Kwon, S. E. \& Chapman, E. R. Doc2 is a $\mathrm{Ca}^{2+}$ sensor required for asynchronous neurotransmitter release. Cell 147, 666-677 (2011).

17. Zhang, Z., Hui, E., Chapman, E. R. \& Jackson, M. B. Regulation of exocytosis and fusion pores by synaptotagmin-effector interactions. Mol. Biol. Cell 21, 2821-2831 (2010).

18. Zhang, X. D., Kim-Miller, M. J., Fukuda, M., Kowalchyk, J. A. \& Martin, T. F. J. $\mathrm{Ca}^{2+}$-dependent synaptotagmin binding to SNAP-25 is essential for $\mathrm{Ca}^{2+}$. triggered exocytosis. Neuron 34, 599-611 (2002).

19. Mohrmann, R. et al. Synaptotagmin interaction with SNAP-25 governs vesicle docking, priming, and fusion triggering. J. Neurosci. 33, 14417-14430 (2013).

20. Earles, C. A., Bai, J. H., Wang, P. \& Chapman, E. R. The tandem C2 domains of synaptotagmin contain redundant $\mathrm{Ca}^{2+}$ binding sites that cooperate to engage t-SNAREs and trigger exocytosis. J. Cell Biol. 154, 1117-1123 (2001).

21. Brose, N., Petrenko, A. G., Sudhof, T. C. \& Jahn, R. Synaptotagmin: a calcium sensor on the synaptic vesicle surface. Science 256, 1021-1025 (1992).

22. Chapman, E. R. \& Davis, A. F. Direct interaction of a $\mathrm{Ca}^{2+}$-binding loop of synaptotagmin with lipid bilayers. J. Biol. Chem. 273, 13995-14001 (1998).

23. Bai, J., Tucker, W. C. \& Chapman, E. R. PIP2 increases the speed of response of synaptotagmin and steers its membrane-penetration activity toward the plasma membrane. Nat. Struct. Mol. Biol. 11, 36-44 (2004).

24. Chicka, M. C., Hui, E. F., Liu, H. S. \& Chapman, E. R. Synaptotagmin arrests the SNARE complex before triggering fast, efficient membrane fusion in response to $\mathrm{Ca}^{2+}$. Nat. Struct. Mol. Biol. 15, 827-835 (2008).

25. Martens, S., Kozlov, M. M. \& McMahon, H. T. How synaptotagmin promotes membrane fusion. Science 316, 1205-1208 (2007).

26. Hui, E. F., Johnson, C. P., Yao, J., Dunning, F. M. \& Chapman, E. R. Synaptotagmin-mediated bending of the target membrane is a critical step in $\mathrm{Ca}^{2+}$-regulated fusion. Cell 138, 709-721 (2009).

27. Lin, C. C. et al. Control of membrane gaps by synaptotagmin- $\mathrm{Ca}^{2+}$ measured with a novel membrane distance ruler. Nat. Commun 5, 5859 (2014).

28. Bhalla, A., Tucker, W. C. \& Chapman, E. R. Synaptotagmin isoforms couple distinct ranges of $\mathrm{Ca}^{2+}, \mathrm{Ba}^{2+}$, and $\mathrm{Sr}^{2+}$ concentration to SNARE-mediated membrane fusion. Mol. Biol. Cell 16, 4755-4764 (2005). 
29. Sutton, R. B., Ernst, J. A. \& Brunger, A. T. Crystal structure of the cytosolic C2A-C2B domains of synaptotagmin III. Implications for $\mathrm{Ca}^{2+}$-independent snare complex interaction. J. Cell Biol. 147, 589-598 (1999).

30. Arac, D. et al. Close membrane-membrane proximity induced by $\mathrm{Ca}^{2+}$ dependent multivalent binding of synaptotagmin-1 to phospholipids. Nat. Struct. Mol. Biol. 13, 209-217 (2006).

31. Fuson, K. L., Montes, M., Robert, J. J. \& Sutton, R. B. Structure of human synaptotagmin $1 \mathrm{C} 2 \mathrm{AB}$ in the absence of $\mathrm{Ca}^{2+}$ reveals a novel domain association. Biochemistry 46, 13041-13048 (2007).

32. Huang, H. \& Cafiso, D. S. Conformation and membrane position of the region linking the two $\mathrm{C} 2$ domains in synaptotagmin 1 by site-directed spin labeling. Biochemistry 47, 12380-12388 (2008).

33. Williamson, M. P. The structure and function of proline-rich regions in proteins. Biochem. J. 297 (Pt 2) 249-260 (1994).

34. Stryer, L. \& Haugland, R. P. Energy transfer: a spectroscopic ruler. Proc. Natl Acad. Sci. USA 58, 719-726 (1967).

35. Hanson, J. A., Brokaw, J., Hayden, C. C., Chu, J. W. \& Yang, H. Structural distributions from single-molecule measurements as a tool for molecular mechanics. Chem. Phys. 396, 61-71 (2012).

36. Schuler, B., Lipman, E. A., Steinbach, P. J., Kumke, M. \& Eaton, W. A. Polyproline and the "spectroscopic ruler" revisited with single-molecule fluorescence. Proc. Natl Acad. Sci. USA 102, 2754-2759 (2005).

37. Doose, S., Neuweiler, H., Barsch, H. \& Sauer, M. Probing polyproline structure and dynamics by photoinduced electron transfer provides evidence for deviations from a regular polyproline type II helix. Proc. Natl Acad. Sci. USA 104, 17400-17405 (2007).

38. Paddock, B. E., Striegel, A. R., Hui, E., Chapman, E. R. \& Reist, N. E. $\mathrm{Ca}^{2+}$-dependent, phospholipid-binding residues of synaptotagmin are critical for excitation-secretion coupling in vivo. J. Neurosci. 28, 7458-7466 (2008)

39. Hui, E., Bai, J. \& Chapman, E. R. $\mathrm{Ca}^{2+}$-triggered simultaneous membrane penetration of the tandem C2-domains of synaptotagmin I. Biophys. J. 91, 1767-1777 (2006)

40. Rosenmund, C. \& Stevens, C. F. Definition of the readily releasable pool of vesicles at hippocampal synapses. Neuron 16, 1197-1207 (1996)

41. Liu, H., Dean, C., Arthur, C. P., Dong, M. \& Chapman, E. R. Autapses and networks of hippocampal neurons exhibit distinct synaptic transmission phenotypes in the absence of synaptotagmin I. J. Neurosci. 29, 7395-7403 (2009).

42. Mansoor, S. E., DeWitt, M. A. \& Farrens, D. L. Distance mapping in proteins using fluorescence spectroscopy: the tryptophan-induced quenching (TrIQ) method. Biochemistry 49, 9722-9731 (2010).

43. Gaffaney, J. D., Dunning, F. M., Wang, Z., Hui, E. \& Chapman, E. R. Synaptotagmin C2B domain regulates $\mathrm{Ca}^{2+}$-triggered fusion in vitro: critical residues revealed by scanning alanine mutagenesis. J. Biol. Chem. 283, 31763-31775 (2008)

44. Herrick, D. Z., Sterbling, S., Rasch, K. A., Hinderliter, A. \& Cafiso, D. S. Position of synaptotagmin I at the membrane interface: cooperative interactions of tandem C2 domains. Biochemistry 45, 9668-9674 (2006).

45. Seven, A. B., Brewer, K. D., Shi, L., Jiang, Q. X. \& Rizo, J. Prevalent mechanism of membrane bridging by synaptotagmin-1. Proc. Natl Acad. Sci. USA 110, E3243-E3252 (2013).

46. Bai, J. H., Tucker, W. C. \& Chapman, E. R. PIP2 increases the speed of response of synaptotagmin and steers its membrane-penetration activity toward the plasma membrane. Nat. Struct. Mol. Biol. 11, 36-44 (2004).

47. Perin, M. S., Fried, V. A., Mignery, G. A., Jahn, R. \& Sudhof, T. C. Phospholipid binding by a synaptic vesicle protein homologous to the regulatory region of protein kinase C. Nature 345, 260-263 (1990).

48. Desai, R. C. et al. The C2B domain of synaptotagmin is a $\mathrm{Ca}^{2+}$-sensing module essential for exocytosis. J. Cell Biol. 150, 1125-1136 (2000).

49. Ho, S. N., Hunt, H. D., Horton, R. M., Pullen, J. K. \& Pease, L. R. Site-directed mutagenesis by overlap extension using the polymerase chain-reaction. Gene 77, 51-59 (1989).

50. Dean, C. et al. Synaptotagmin-IV modulates synaptic function and long-term potentiation by regulating BDNF release. Nat. Neurosci. 12, 767-776 (2009).
51. Matthew, W. D., Tsavaler, L. \& Reichardt, L. F. Identification of a synaptic vesicle-specific membrane protein with a wide distribution in neuronal and neurosecretory tissue. J. Cell Biol. 91, 257-269 (1981).

52. Bolte, S. \& Cordelieres, F. P. A guided tour into subcellular colocalization analysis in light microscopy. J. Microsc. Oxf. 224, 213-232 (2006).

53. Phillips, J. C. et al. Scalable molecular dynamics with NAMD. J. Comput. Chem. 26, 1781-1802 (2005).

54. MacKerell, A. D. et al. All-atom empirical potential for molecular modeling and dynamics studies of proteins. J. Phys. Chem. B 102, 3586-3616 (1998).

55. Mackerell, A. D., Feig, M. \& Brooks, C. L. Extending the treatment of backbone energetics in protein force fields: Limitations of gas-phase quantum mechanics in reproducing protein conformational distributions in molecular dynamics simulations. J. Comput. Chem. 25, 1400-1415 (2004).

56. Laio, A. \& Gervasio, F. L. Metadynamics: a method to simulate rare events and reconstruct the free energy in biophysics, chemistry and material science. Rep. Prog. Phys. 71, 126601 (22pp) (2008).

57. Fischer, S., Dunbrack, R. L. \& Karplus, M. Cis-trans imide isomerization of the proline dipeptide. J. Am. Chem. Soc. 116, 11931-11937 (1994).

58. Brooks, B. R. et al. CHARMM: the biomolecular simulation program. J. Comput. Chem. 30, 1545-1614 (2009).

59. Xu, J., Mashimo, T. \& Sudhof, T. C. Synaptotagmin-1,-2, and-9: $\mathrm{Ca}^{2+}$ sensors for fast release that specify distinct presynaptic properties in subsets of neurons. Neuron 54, 567-581 (2007).

\section{Acknowledgements}

We thank J. Gaffaney and C. Evans for help analysing the linker mutants. This study was supported by a grant from the National Institutes of Health (MH 61876 to E.R.C.). The computational work was supported by the University of Wisconsin Materials Research Science and Engineering Center (DMR-1121288) through a SEED grant to Q.C. and A.Y., and by an NSF instrumentation grant (CHE-0840494) awarded to the Department of Chemistry, and by the Extreme Science and Engineering Discovery Environment (XSEDE), also from NSF (OCI-1053575). E.R.C. is an Investigator of the Howard Hughes Medical Institute.

\section{Author contributions}

H. Bai and H. Bao performed the biochemical experiments; R.X. performed the electrophysiological experiments; L.Z., A.Y. and Q.C., designed the simulation studies; L.Z. conducted the simulations and L.Z., A.Y. and Q.C. analysed the simulation data; E.R.C., H. Bai and R.X. designed and analysed all of the biochemistry and physiology data, and co-wrote the paper.

\section{Additional information}

Supplementary Information accompanies this paper at http://www.nature.com/ naturecommunications

Competing financial interests: The authors declare no competing financial interests.

Reprints and permission information is available online at http://npg.nature.com/ reprintsandpermissions/

How to cite this article: Bai, H. et al. Different states of synaptotagmin regulate evoked versus spontaneous release. Nat. Commun. 7:10971 doi: 10.1038/ncomms10971 (2016).

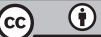

This work is licensed under a Creative Commons Attribution 4.0 International License. The images or other third party material in this article are included in the article's Creative Commons license, unless indicated otherwise in the credit line; if the material is not included under the Creative Commons license, users will need to obtain permission from the license holder to reproduce the material. To view a copy of this license, visit http://creativecommons.org/licenses/by/4.0/ 